\title{
Técnicas de controle e métodos de sincronização para sistemas de energia renovável ligados à rede elétrica - Uma revisão
}

\section{Control techniques and synchronization methods for grid-connected renewable energy systems - A survey}

\author{
Sebastián Manrique Machado ${ }^{1}$; Newton Da Silva ${ }^{2}$
}

\section{Resumo}

Os Sistemas de Energia Renovável (SER) tornaram-se uma alternativa atrativa frente às fontes de energia convencional, devido à necessidade de diversificar a matriz energética e assim garantir o abastecimento da demanda futura de eletricidade de uma forma sustentável. As fontes renováveis precisam de equipamentos de conversão de energia com um controle adequado para conseguir operar ligados à rede elétrica de maneira satisfatória. $\mathrm{O}$ controle deve garantir a sincronização com a rede e o fornecimento de corrente senoidal para cumprir com as normas internacionais de qualidade de energia. Este trabalho apresenta de forma geral as principais técnicas de controle de corrente para os SER e os métodos de sincronização com a rede elétrica, apresentados na literatura. Técnicas de controle linear e não linear são abordadas, considerando diferentes sistemas de referência, assim como métodos de sincronização monofásicos e trifásicos.

Palavras-chave: Energias renováveis, controle de corrente, inversor ligado à rede, PLL.

\begin{abstract}
Renewable Energy Systems have become an attractive alternative to the conventional energy sources, due the necessity of energetic matrix diversification in order to ensure the supply of the future electricity demand in a sustainable way. The renewable sources need energy conversion equipment with suitable control in order to achieve a satisfactory grid-tied operation. The control must ensure the synchronization with the grid and the sinusoidal current injection in order to meet the international power quality standards. This work provides an overview of the main current control strategies for Renewable Energy Systems, and grid synchronization methods presented in the literature. Linear and non-linear current control techniques are covered regarding different reference frames, and single phase and three phase synchronization methods are presented as well.
\end{abstract}

Keywords: Renewable sources, current control, grid-connected inverter, PLL.

\footnotetext{
${ }^{1}$ Docente da Coordenação de Engenharia Elétrica, Universidade Tecnológica Federal Do Paraná Campus Apucarana- COELTUTFPR; sebastiand@utfpr.edu.br

${ }^{2}$ Docente do Departamento de Engenharia Elétrica da Universidade Estadual de Londrina - DeEL-UEL; newton.silva@uel.br
} 


\section{Introdução}

$\mathrm{Na}$ atualidade, os SER representam uma oportunidade para garantir o fornecimento de energia elétrica a médio e longo prazo de uma forma sustentável com o meio ambiente, reduzindo a dependência das fontes convencionais, especialmente dos combustíveis fósseis, e diversificando a matriz energética dos países.

Para fornecer energia à rede elétrica por meio de painéis fotovoltaicos ou geradores eólicos modernos, precisa-se de uma etapa de acondicionamento desta energia, o qual é feito por equipamento baseado em eletrônica de potência, cujas principais tarefas incluem a maximização da extração de energia das fontes renováveis e o cumprimento com requisitos de tensão, frequência e qualidade da energia (TEODORESCU; LISERRE; RODRIGUEZ, 2011). Em qualquer caso, o inversor de potência é o último equipamento a fazer interface entre as fontes renováveis e a rede elétrica.

Na Figura 1, é apresentada a estrutura geral de um SER baseado em um inversor alimentado por tensão (Voltage Source Inverter - VSI). Neste caso, a fonte de corrente $\left(I_{R}\right)$ representa a injeção de potência no barramento $\mathrm{CC}$ proveniente das fontes renováveis, seja por conexão direta ou por parte de um estágio de conversão prévio. O controle consiste nos compensadores em cascata externo e interno. $\mathrm{O}$ compensador externo regula a tensão do barramento $\mathrm{CC}$ para obter um equilíbrio entre a potência absorvida elo capacitor e a fornecida para a rede elétrica. Posteriormente, encontra-se o controlador interno de corrente, o qual recebe um sinal de referência proveniente do compensador da tensão do barramento $\mathrm{CC}$, e um sinal de sincronismo proveniente do PLL (Phase Locked Loop), que serve como referência para fornecer uma corrente em fase com a tensão da rede. Assim, o inversor opera como uma fonte de corrente controlada pela tensão do barramento CC. Caso seja necessário fornecer ou absorver potência reativa da rede, o controlador de corrente receberá outro sinal de referência como se pode observar na Figura 1.
Finalmente, o filtro atenua as componentes de corrente e tensão nas frequências de chaveamento do inversor (BACHA; MUNTEANU; BRATCU, 2014). Neste trabalho a rede elétrica é desenhada de forma unifilar, podendo representar tanto sistemas trifásicos quanto monofásicos.

Figura 1 - Diagrama geral de um SER e seu controle.

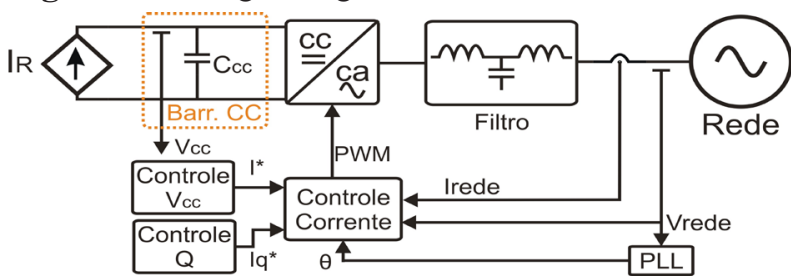

Fonte: Os autores

Devido ao controlador de corrente ser responsável tanto pela qualidade da energia fornecida à rede, como pela estabilidade do sistema, é fundamental garantir seu adequado desempenho para conseguir uma satisfatória integração dos SER com a rede. Atualmente, os SER devem cumprir com normas internacionais, como a IEC 61727 e IEEE 1547, as quais fixam limites de injeção de correntes harmônicas e da Taxa de Distorção Harmônica (TDH). Porém, as normas cada vez se tornarão mais exigentes e as funcionalidades, como o suporte de controle de potência reativa, o de regulação de frequência ativa em função da frequência e a capacidade de operar mesmo com a rede em falha, provavelmente serão exigências nas regulações futuras (YANG, YONGHENG; WANG; BLAABJERG, 2014; YANG, YONGHENG et al., 2013). Isto tem motivado muitas pesquisas recentes em relação às técnicas e estruturas para o controle da corrente. Do mesmo modo, como o funcionamento do controlador de corrente está determinado pelo desempenho do PLL, então este também tem sido um alvo de estudo para os pesquisadores. Neste trabalho, apresenta-se uma revisão tanto das técnicas de controle de corrente quanto dos tipos de PLL apresentados na literatura.

O trabalho aborda inicialmente as técnicas de controle de corrente lineares e não lineares, e posteriormente diferentes técnicas empregadas para fornecer o sinal de sincronismo. 


\section{Técnicas de controle linear}

O controle dos inversores apresenta a necessidade de rastrear um sinal de referência senoidal, fato que se torna mais relevante na hora de aplicar as técnicas de controle linear, pois o tradicional controle ProporcionalIntegral (PI) não consegue eliminar o erro de fase, nem de magnitude em regime permanente. Portanto, é necessário aplicar transformações de coordenadas para utilizar este controlador ou, caso contrário, desenvolver outros tipos de compensadores. Nesta seção serão apresentadas diferentes estratégias de controle linear baseadas na aplicação do compensador PI, do Proporcional-Ressonante (PR) e do Controle Repetitivo (CR). Nesta seção serão apresentadas diferentes estratégias de controle linear baseadas na aplicação do compensador PI, do Proporcional-Ressonante (PR) e do Controle Repetitivo (CR).

O controle no sistema $d-q$ tem sido amplamente estudado e aplicado ao controle de corrente em SER ligados à rede elétrica (DASH; YAZDANI, 2008; SACCOMANDO; SVENSSON, 2001; TWINING; HOLMES, 2003).

A técnica consiste em utilizar a transformação apresentada na Equação (1) ou transformação $a b c-d q 0$, a qual muda de um sistema de referência estacionário para um sistema de

Figura 2 - Controle no sistema $d$ - $q$ com sistema de desacoplamento entre eixos e feed-forfard de tensão.

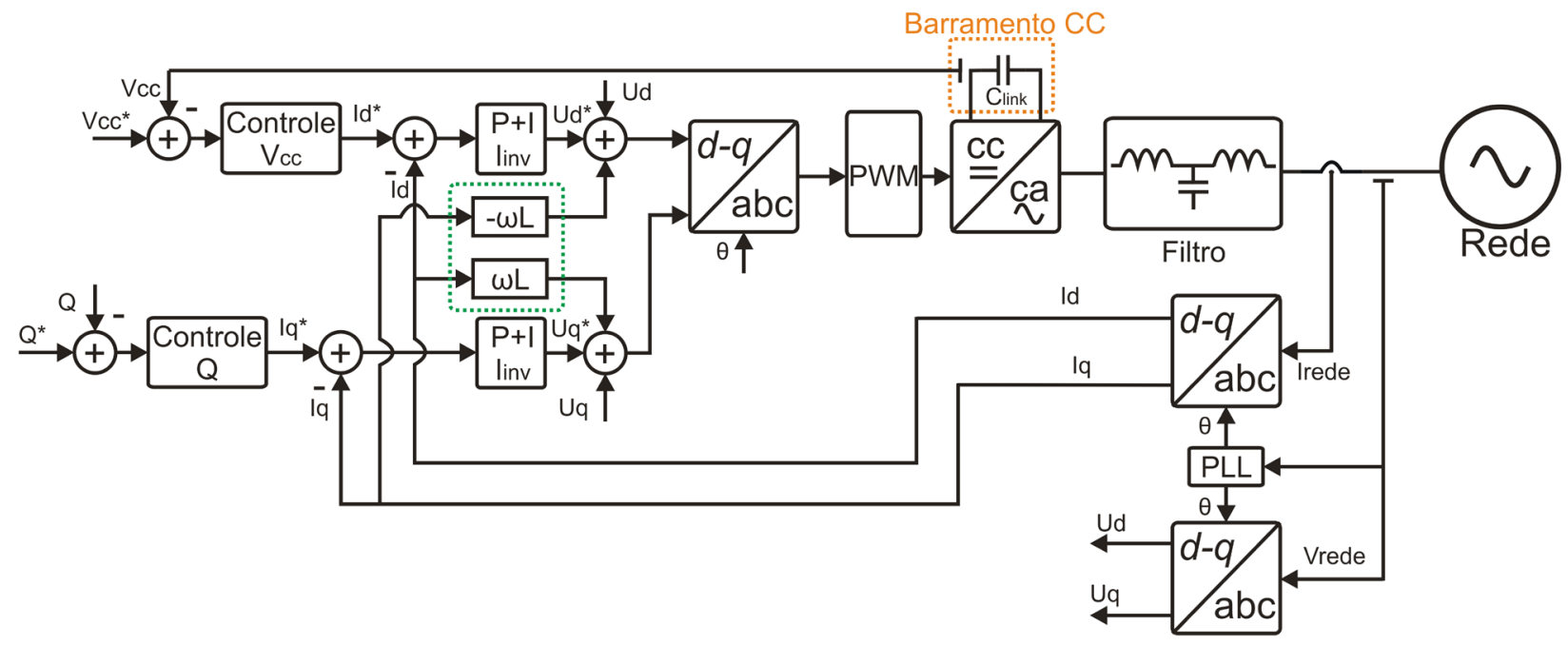

Fonte: Os Autores

referência em sincronismo com a frequência da rede. Como resultado, o sistema trifásico de sinais alternados torna-se um sistema ortogonal de sinais contínuos, fato que facilita a aplicação do controlador PI clássico(TIMBUS et al., 2009) .

$$
\left[\begin{array}{l}
d \\
q \\
0
\end{array}\right]=\frac{2}{3}[T]\left[\begin{array}{l}
a \\
b \\
c
\end{array}\right]
$$

onde $T$ é definida na Equação (2)

$$
[T]=\left[\begin{array}{ccc}
\cos \theta & \cos \left(\theta-\frac{2 \pi}{3}\right) & \cos \left(\theta+\frac{2 \pi}{3}\right) \\
-\operatorname{sen} \theta & -\operatorname{sen}\left(\theta-\frac{2 \pi}{3}\right) & -\operatorname{sen}\left(\theta+\frac{2 \pi}{3}\right) \\
1 / 2 & 1 / 2 & 1 / 2
\end{array}\right]
$$

A Figura 2 apresenta a estrutura do controle no sistema $d-q$. A realimentação de estado (termos $\omega L$ ) é usada para desacoplar o controle das correntes $i_{d}$ $\mathrm{e}^{i} q$, enquanto que o feed-forward de tensão é usado para aumentar a rejeição de perturbações da rede por parte do controle. Recentemente, estudos sobre outras técnicas de desacoplamento do controle das corretes no sistema $d-q$ têm sido utilizadas na literatura, com o objetivo de aprimorar o desempenho desta estrutura de controle (YEPES et al., 2014; ZHOU et al., 2015). Mesmo assim, para cumprir com as normas internacionais, é indispensável o uso de 
compensadores de harmônicas cuja implementação no sistema $d-q$ implica um aumento importante da complexidade e da carga computacional, devido à necessidade de filtros adicionais e da aplicação da transformação da Equação (1) para cada uma das frequências que irão ser compensadas (BLAABJERG et al., 2006; TIMBUS et al., 2009).

Uma alternativa ao controle de corrente no sistema $d-q$ foi proposta por Yuan et al. (2002) e Zmood e Holmes (2003), que consiste em generalizar o conceito do controlador PI, o qual elimina o erro de regime permanente para sinais contínuos, pois possui um polo em zero e, portanto, o ganho deste controlador em $f=0 \mathrm{~Hz}$ é teoricamente infinito. O controlador $\mathrm{PR}$, cuja função de transferência é apresentada na Equação (3), oferece um ganho teoricamente infinito na frequência $\omega_{o}$, sendo, portanto, adequado para rastrear sinais alternados próprios dos sistemas $\alpha-\beta$ e $a b c$. Em Teodorescu et al. (2006) pode-se consultar a equivalência matemática que existe entre o controlador PI no sistema $d-q$ e o controlador PR no sistema $\alpha-\beta$, onde a parte ressonante pode ser considerada como um integrador de sinais alternados (TEODORESCU; BLAABJERG, 2004b), conceito que deu origem ao termo SOGI (Second Order Generalized Integrator).

$$
P R(s)=k_{p}+\frac{2 k_{i} s}{s^{2}+\omega_{o}^{2}}
$$

A Figura 4 apresenta a estrutura geral do controle no sistema $\alpha-\beta$. Observa-se que é necessário o bloco da transformação do sistema d-q para o sistema $\alpha-\beta$ no caso de se controlar a potência reativa. Caso contrário, o bloco pode ser substituído por um bloco cujo sinal de saída é senoidal em fase com a tensão da rede elétrica, e a amplitude é controlada pelo regulador de tensão do barramento CC. Em sistemas trifásicos para a realimentação das correntes, o uso da transformação de Clarke na Equação (4) é necessária, a qual precisa de menos recursos computacionais que a transformação na Equação (1), uma vez que não envolve cálculos com funções trigonométricas.

$$
\left[\begin{array}{l}
\alpha \\
\beta \\
0
\end{array}\right]=\frac{2}{3}\left[\begin{array}{ccc}
1 & -1 / 2 & -1 / 2 \\
0 & \sqrt{3} / 2 & -\sqrt{3} / 2 \\
1 / 2 & 1 / 2 & 1 / 2
\end{array}\right]\left[\begin{array}{l}
a \\
b \\
c
\end{array}\right]
$$

Uma das vantagens mais significativas do controlador PR é a facilidade de se implementar um Compensador de Harmônicas $(\mathrm{CH})$. Basta adicionar blocos ressonantes em paralelo sintonizados nas frequências harmônicas a ser compensadas, como é ilustrado na Figura 4. Devido a atuação de cada bloco ressonante ser somente para um valor de frequência, a compensação seletiva de harmônicas não afeta a dinâmica do controle da fundamental (TEODORESCU; BLAABJERG, 2004a; YUAN et al., 2002).

Em relação à implementação prática, a ressonância do controle é amortecida para evitar problemas de estabilidade do inversor e de saturação numérica do processador, dando preferência à implementação da função de transferência na Equação (5), a qual pode ser decomposta em blocos básicos conforme a Figura 3. Diferentes implementações do controlador ressonante foram comparadas por (BOSIO et al., 2015) .

$$
R(s)=\frac{2 k_{i} \zeta \omega_{o} s}{s^{2}+2 \zeta \omega_{o} s+\omega_{o}^{2}} ; \operatorname{com} \zeta \omega_{o}=\omega_{c}
$$

Figura 3 - Decomposição do controle PR em blocos básicos.

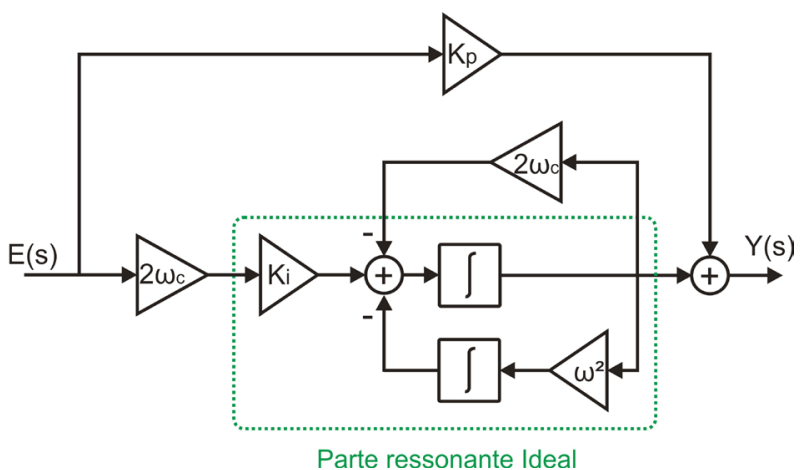

Fonte: Os autores 
Figura 4 - Estrutura do controle no sistema $\alpha-\beta$.

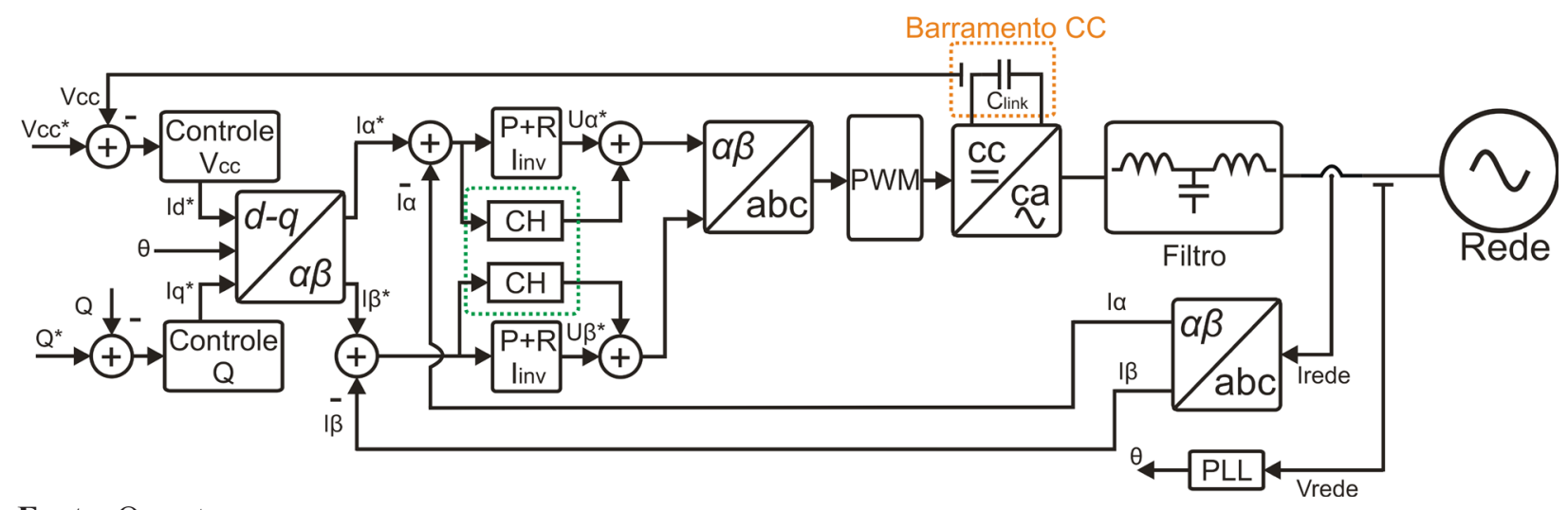

Fonte: Os autores

A implementação digital do controlador PR feita através da transformação bilinear ou de Tustin, tem sido amplamente utilizada na literatura (CASTILLA et al., 2009; GAZOLI et al., 2013; NASCIMENTO et al., 2014).

Porém, quando são usadas altas taxas de amostragem e processadores de ponto fixo, os erros de arredondamento produzem um deslocamento indesejado das frequências de ressonância. Para estes casos, a técnica de digitalização baseada no operador delta deve ser utilizada (NEWMAN; HOLMES, 2003; SERA et al., 2005). Os efeitos das diferentes técnicas de discretização do controlador no desempenho do sistema têm sido analisados em (YEPES et al., 2010; ZHANG; SPENCER; GUERRERO, 2013). Para ajustar os parâmetros do controle PR, encontram-se diferentes metodologias. Algumas destas são técnicas baseadas na teoria polinomial de Naslim (DUMITRESCU et al., 2007), na alocação direta de polos (LI et al., 2012), na resposta em frequência (NASCIMENTO et al., 2014), na extrapolação da metodologia tradicional do ajuste de controladores PI (TEODORESCU; BLAABJERG, 2004a; YUAN et al., 2002), na análise dos diagramas de Nyquist e funções de sensibilidade (YEPES et al., 2011a), e na relação entre a frequência de amostragem e a frequência de ressonância do filtro da saída do inversor (PARKER; MCGRATH; HOLMES, 2014) . Outra opção para a implementação do $\mathrm{CH}$ na estrutura de controle no sistema $\alpha-\beta$, é utilizar o $\mathrm{CR}$, cuja principal vantagem é que só necessita de um retardo para compensar todas as harmônicas. Duas alternativas de implementação são apresentadas na Figura 5, onde $\mathrm{T}_{\mathrm{d}}$ representa o período do sinal alternado da primeira harmônica a compensar. A implementação da Figura 5a, faz a realimentação e ofeed-forward positivamente, compensando tanto harmônicas pares quanto ímpares, segundo sua função de transferência na Equação (6). Na Figura 5b, é ilustrada uma implementação com realimentação e feed-forward negativo, sendo somente compensadas harmônicas ímpares, conforme a sua função de transferência na Equação (7). 
Figura 5 - Duas alternativas para implementar o $\mathrm{CH}$ usando o CR.

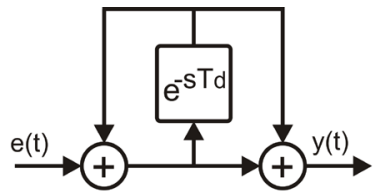

a) positivo

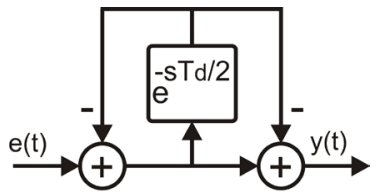

b) negativo
Fonte: Os autores baseado em Teodorescu et al. (2006).

$$
\begin{aligned}
& \frac{Y(s)}{E(s)}=\frac{1+e^{-s T_{d}}}{1-e^{-s T_{d}}}=\frac{2}{T_{d}}\left\{\frac{1}{s}+\sum_{h=1}^{\infty} \frac{2 s}{s^{2}+(h \omega)^{2}}\right\} \\
& \frac{Y(s)}{E(s)}=\frac{1-e^{-s T_{d} / 2}}{1+e^{-s T_{d} / 2}}=\frac{4}{T_{d}}\left\{\sum_{k=1}^{\infty} \frac{2 s}{s^{2}+((2 k-1) \omega)^{2}}\right\}
\end{aligned}
$$

Outros tipos de implementações do $\mathrm{CH}$, utilizando o CR, também podem ser consultadas (COSTACASTELLÓ; GRINÓ; FOSSAS, 2004; LIU et al., 2012; MASTROMAURO et al., 2009).

Devido ao fato de pequenos desvios do valor nominal da frequência puderem afetar consideravelmente o desempenho dos controladores ressonantes (PR e $\mathrm{CR}$ ), recentemente vários esforços para conseguir implementações adaptativas com a frequência da rede têm sido apresentados (YANG, YONGHENG; ZHOU; BLAABJERG, 2015). Basicamente, existem duas tendências. A primeira é a utilização da teoria de filtros digitais adaptativos, empregando o algoritmo Least Mean Square (LMS) (BLASKO, 2007), a recursão Schur e a estrutura Lattice (GONZALEZ-ESPIN et al., 2012, 2013), ou fazendo a adaptação digital da estrutura de dois integradores, apresentada na Figura 3 (YEPES et al., 2011b). A segunda tendência consiste na implementação digital adaptativa do CR, na qual existem duas opções, a adaptação do número de amostras que o buffer retarda (CHEN, DONG; ZHANG; QIAN, 2013; NAZIR et al., 2014; YANG, Y; ZHOU; BLAABJERG, 2016) ou a adaptação da frequência de amostragem proposta por (ZANCHETTA; SHARKH; ABUSARA, 2015).

\section{Técnicas de controle não linear}

As técnicas de controle não linear, em geral, possuem uma dinâmica mais rápida, normalmente o suficiente para eliminar as preocupações com a injeção de harmônicas de baixa ordem. Portanto, estes controladores não precisam do uso do $\mathrm{CH}$ (BLAABJERG et al., 2006). A estrutura de controle é a mesma apresentada na Figura 4, sendo somente substituído o bloco PR pelo bloco de controle não linear, apresentado em cada caso.

O controle de corrente por histerese ou HCC (Hysteresis Current Control) é uma das estratégias mais simples, onde a corrente alternada é controlada para mantê-la dentro de um limite superior e um inferior, ou banda de histerese, entorno de uma corrente senoidal de referência, conforme as Figuras 6a e 6b. A saída deste controlador representa os estados das chaves, o que implica que não há modulação PWM (MONFARED; GOLESTAN, 2012). Como consequência, a frequência de comutação não é constante, fato que dificulta o projeto do filtro de saída e torna-se uma das maiores desvantagens desta técnica. Uma frequência de comutação constante pode ser obtida ajustando de forma adaptativa a banda de histerese, porém sua implementação pode requerer uma circuitaria analógica considerável (HO; CHEUNG; CHUNG, 2009; MALESANI; MATTAVELLI, 1997).

Recentemente, com o uso de processadores com maior capacidade de processamento, implementações adaptativas totalmente digitais deste controlador têm sido realizadas (DAVOODNEZHAD; HOLMES; MCGRATH, 2014). Uma versão desta técnica de controle adaptada para o sistema $d-q$ e com modulação Space-Vector PWM para sistemas trifásicos foi apresentada por (SUUL, JON ARE et al., 2011). Neste caso os sinais de referência e a banda de histerese são valores constantes, fato que simplifica ainda mais sua implementação. 
Figura 6 - Controle HCC. a) Diagrama de blocos. b) Sinal de corrente de saída.

a) Diagrama de Blocos HCC

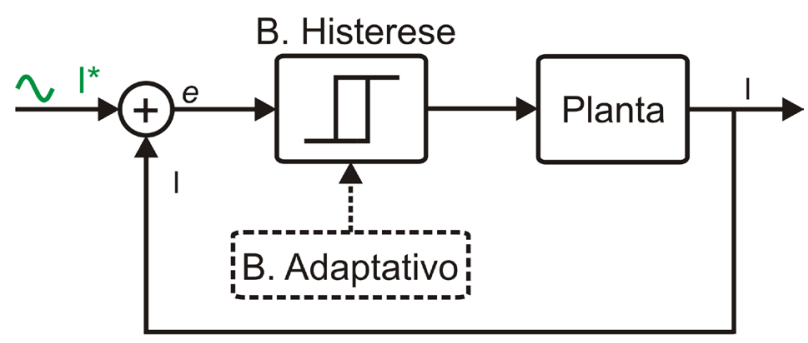

b) Sinal de corrente HCC

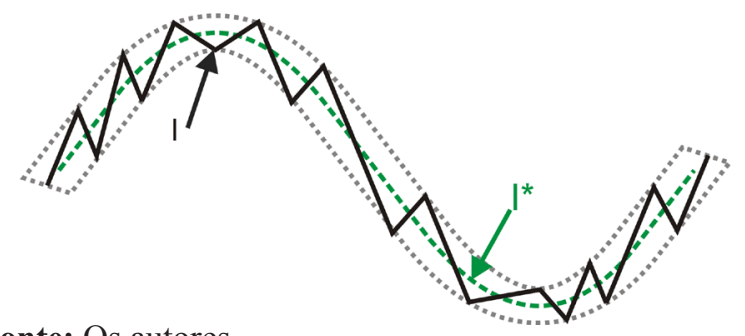

Fonte: Os autores

O controlador Dead-Beat é uma das técnicas clássicas da teoria de controle em tempo discreto. Trata-se de uma técnica baseada no modelo, que consiste em sintetizar o sinal de entrada que deve ser aplicado a um sistema para que o erro seja igual a zero. Porém, devido aos efeitos da amostragem e cálculos computacionais, este controlador introduz um retardo equivalente a um período de amostragem. Uma implementação digital deste controlador pode ser feita conforme a Equação (8) (TIMBUS et al., 2009).

$$
G_{D B}(z)=\left(\frac{1}{b}\right) \frac{1-a z^{-1}}{1-Z^{-1}}
$$

onde os parâmetros $a$ e $b$ são definidos em (9) e (10), respectivamente.or pode ser feita conforme a Equação (8) (TIMBUS et al., 2009).

$$
\begin{gathered}
a=e^{-\left(\frac{R_{T}}{L_{T}}\right) T_{S}} \\
b=-\frac{1}{R_{T}}\left(e^{-\left(\frac{R_{T}}{L_{T}}\right) T_{S}}-1\right)
\end{gathered}
$$

sendo $\mathbf{R}_{\mathbf{T}}$ e $\mathbf{L}_{\mathbf{T}}$ a resistência e a indutância total do filtro.

As Equações (9) e (10) deixam em evidência dependência do controlador em relação aos parâmetros da planta e, em consequência, o sistema não é robusto, o qual constitui o maior inconveniente deste tipo de controlador.

Figura 7 - Diagrama genérico do controlador DeadBeat.

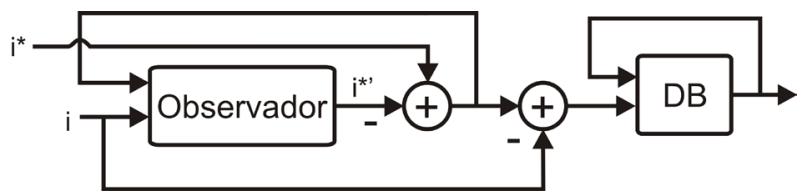

Fonte: os autores

Tanto o retardo introduzido pelo controlador DeadBeat como sua robustez, podem ser aprimorados através do projeto de observadores utilizando um esquema similar ao apresentado na Figura 7 (MALESANI, 1999; MATTAVELLI, 2005). Mesmo assim, não há muitas pesquisas recentes em relação à aplicação deste tipo de controladores em SER.

A realimentação não linear composta ou CNF (Composite Non-linear Feedback) é uma técnica recentemente aplicada ao controle da corrente de inversores ligados narede elétrica(EREN; BAKHSHAI; JAIN, 2014; EREN, 2013). O método consiste em duas realimentações: um laço de realimentação linear baseada na realimentação de estados, e outro laço de realimentação não linear. A parte linear é projetada para obter uma resposta transitória rápida, com uma baixa relação de amortecimento. A parte não linear é projetada para aumentar o amortecimento na medida em que o sistema aproxima-se à referência (CHEN, B.M. et al., 2003). Assim, esta estratégia de controle consegue que o amortecimento do sistema mude dependendo se está operando em condições transitórias ou de regime permanente. Na Figura 8, é apresentado o diagrama de blocos que descreve o funcionamento desta técnica. 
Figura 8 - Diagrama de blocos do controlador CNF.

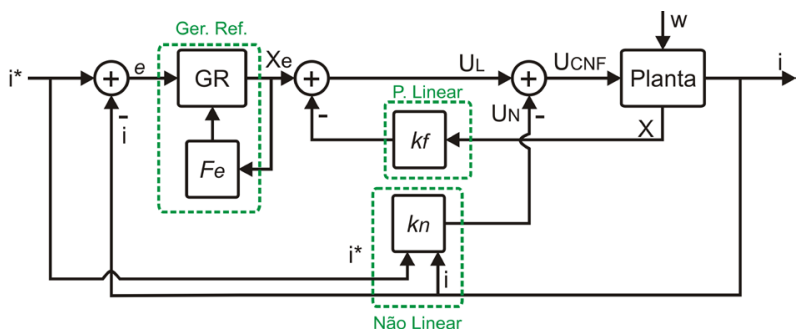

Fonte: Os autores

Observa-se na Figura 8, que a parte linear do controle consiste na clássica alocação de polos por meio da realimentação dos estados da planta. Assim, a matriz de ganhos $k_{f}$, pode ser encontrada com a equação de Ackerman e alocar os polos a fim de obter uma rápida resposta transitória. Além disso, de acordo com o principio do modelo interno IMP (Internal Mode Principle), um bloco Gerador de Referência deve ser adicionado para garantir que o controle possa rastrear sinais senoidais com o mínimo erro em regime permanente. O gerador de referência é projetado também no espaço de estados para alocar dois polos complexos conjugados na frequência da rede e um polo em zero. Finalmente, a parte não linear consiste numa função, geralmente do tipo exponencial, que tem como entradas a referência e o sinal de saída. A função exponencial na Equação (11) foi usada por Eren; Bakhshai; Jain, (2014); e Eren, (2013). Neste caso observa-se que quando a saída é igual à referência, a parte não linear torna-se um simples ganho igual a $k_{1}$; e se a referência for muito maior que a saída do sistema, então a parte linear não atua, deixando o sistema com um baixo amortecimento.

$$
k_{n}=\frac{k_{1}}{1-e^{-1}}\left(e^{-\left(1-\frac{i}{i *}\right)}-e^{-1}\right)
$$

O CNF é uma estratégia que melhora significativamente a resposta transitória do sistema, porém é uma estrutura mais complexa e que pode precisar do projeto de observadores de estado.

\section{Métodos de sincronização com a rede}

O desempenho do controlador de corrente em SER ligados à rede elétrica está altamente influenciado pelo desempenho do sistema que gera o sinal de sincronismo. As informações de fase, frequência, e amplitude da tensão da rede são críticas e precisase de algoritmos confiáveis e robustos que possam providenciar essas informações com a melhor exatidão a precisão possível, além de rapidez.

Diversas estratégias de sincronização têm sido apresentadas na literatura, algumas baseadas na detecção do cruzamento por zero ou em filtros para os sinais de tensão. Porém, na atualidade a técnica do Phase Locked Loop (PLL) é a mais empregada, devido a ser um sistema em malha fechada que monitora constantemente a tensão da rede. Um diagrama genérico de um PLL é apresentado na Figura 9, o qual é composto por um bloco detector de fase, um controlador, que geralmente é um PI, e finalmente há um oscilador controlado por tensão ou VCO (VoltageControlled Oscilator) (YANG, YONGHENG, 2014).

Figura 9 - Diagrama genérico de um PLL.

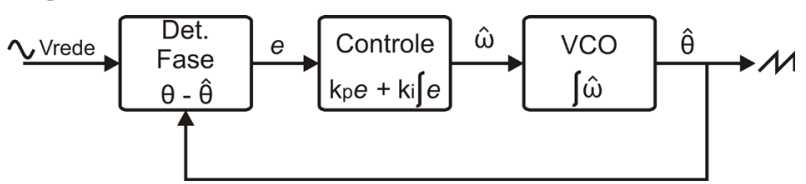

Fonte: Os autores

Uma implementação típica de um PLL trifásico é apresentada na Figura 10, a qual usa a transformação $d$ - $q$ e por tanto é conhecido como SRF-PLL (Syncronous Reference Frame -PLL). O ângulo estimado pelo algoritmo alimenta o bloco da transformação $d-q$, e a tensão do eixo direto $V_{d}$ é realimentado ao controlador PI que tenta mantê-la em zero. Com a finalidade de melhorar o desempenho do PLL em redes distorcidas e com tensões desbalanceadas, diferentes algoritmos e técnicas de filtragem tem sido apresentadas na literatura, para que o PLL opere somente com as componentes de sequência positiva. 
Figura 10 - PLL trifásico usando a transformação $d-q$.

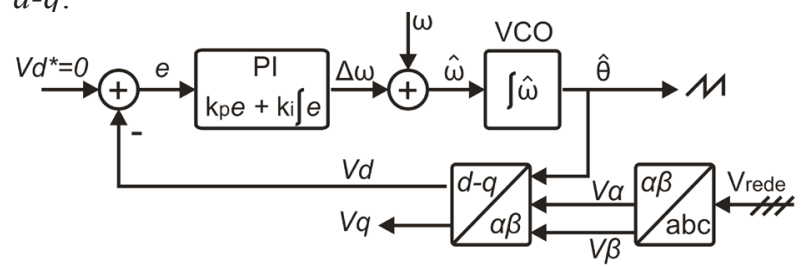

Fonte: Os autores

Algumas técnicas de melhoramento do PLL da Figura 10 são o EPLL (Enhanced PLL) (KARIMIGHARTEMANI; IRAVANI, 2004), a utilização da teoria PQ (SILVA et al., 2006) as técnicas de desacoplamento no sistema $d-q$ (RODRÍGUEZ et al., 2007), ou a aplicação do operador DSC (Delayed Signal Cancelation) (GOLESTAN et al., 2015).

Na Figura 11 ilustra-se uma alternativa ao SRFPLL, a qual está baseada no fato de que o valor médio do produto de dois sinais ortogonais é zero. O método consiste em aplicar uma função trigonométrica ao ângulo estimado e multiplicar o sinal obtido pelo sinal da rede. O controlador PI atuará para que estes sinais sejam ortogonais, consequentemente valor médio do seu produto se aproxime a zero. Embora na Figura 11, apresenta uma implementação monofásica, o conceito facilmente pode ser levado à implementação trifásica através da obtenção de sinas desfasados $120^{\circ}$ e multiplicando-os pelos sinais correspondentes da rede. Posteriormente, o valor médio de cada um destes resultados é somado e realimentado (PÁDUA et al., 2007).

Em relação aos sistemas monofásicos, utilizamse as mesmas estruturas dos sistemas trifásicos, simplesmente deve ser adicionado um bloco gerador

Figura 11 - PLL usando o valor médio do produto de dois sinais ortogonais.

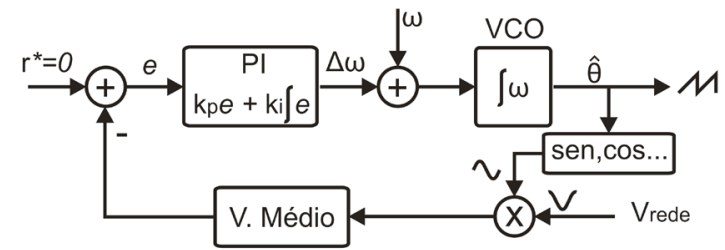

Fonte: Os autores de sinais ortogonais e filtragem. A estratégia utilizada para realizar essas duas funções é a principal diferença entre os diferentes tipos de PLL na literatura.

Na Figura 12a apresenta-se a forma mais simples de gerar os sinais ortogonais, a qual consiste em introduzir um retardo equivalente a um quarto do período do sinal de tensão da rede elétrica. Desta forma não há nenhuma função de filtragem o qual pode afetar o desempenho do PLL (BLAABJERG et al., 2006). O SOGI que foi aplicado no controle PR pode gerar dois sinais ortogonais, e um deles em fase com a rede. Na Figura $12 \mathrm{~b}$, apresenta-se o diagrama de blocos do SOGI como gerador de sinais ortogonais. Esta implementação traz como beneficio que as componentes diferentes da fundamental são filtradas, e por tanto as componentes harmônicas geram menos efeitos negativos sobre o desempenho do PLL (CIOBOTARU; TEODORESCU; BLAABJERG, 2006; YANG, YONGHENG, 2014).

Figura 12 - Alternativas de geração de sinais ortogonais. a). Implementando retardo. b) Utilizando um gerador ortogonal de segunda ordem.

a) Retardo

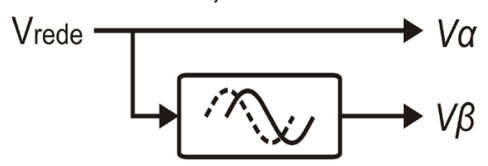

Retardo $\mathrm{T} / 4$

b) SOGI

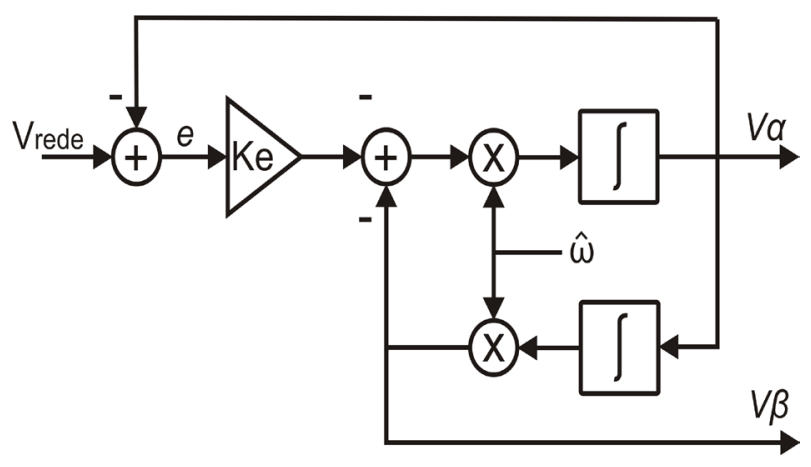

Fonte: Os autores 
Recentemente, diferentes pesquisas têm sido realizadas para melhorar o desempenho dos SER através do aprimoramento do PLL, como a implementação adaptativa com a frequência (AMA et al., 2013; DESTRO et al., 2013), as novas técnicas de filtragem computacionalmente eficientes (GOLESTAN et al., 2015, 2016), a adaptação da largura de banda do PLL segundo a impedância da rede (WU et al., 2015) e o aprimoramento da estabilidade do sistema através da melhora do desempenho do PLL (SUUL et al., 2015).

\section{Conclusões}

Neste trabalho as diferentes estratégias de controle de corrente utilizadas em SER ligados à rede elétrica foram apresentadas. Discutiram-se estratégias tanto de controle linear quanto não linear, considerando sua aplicação no sistema $d-q$ e no sistema $\alpha-\beta$. As principais caraterísticas de cada controlador, sua capacidade para compensar harmônicas de baixa ordem, assim como detalhes referentes à sua implementação prática e ajuste foram também apresentados.

$\mathrm{Na}$ atualidade o controlador PR é o mais utilizado em SER por sua capacidade para compensar harmônicas. Além disso, o controle PR permite ajustar o ganho para cada harmônica, enquanto que o CR não. Em contraposição, as técnicas de controle não lineares possuem uma dinâmica mais rápida e as harmônicas de baixa ordem deixam de ser uma preocupação. Considerando que o controlador Dead-beat proporciona uma baixa robustez, o HCC e o CNF parecem ser as técnicas com caraterísticas mais adequadas para SER. Pesquisas adicionais para conseguir implementações digitais mais eficientes do HCC devem ser feitas. No caso do CNF, precisase de pesquisas que proporcionem informações sobre a sintonização dos seus parâmetros e seus efeitos no desempenho do controlador, assim como análises de seu comportamento frente a úrbios na rede elétrica.
As diferentes técnicas de sincronização com a rede elétrica também foram tratadas, junto com diferentes métodos de filtragem para a extração da componente fundamental. O processo de filtragem se torna cada vez mais necessário devido ao aumento da presença de equipamentos não lineares na rede elétrica, por tanto, melhorar as técnicas de filtragem, assim como sua eficiência computacional, foram identificadas como as necessidades mais urgentes nas em relação às técnicas de sincronização com a rede.

\section{Agradecimentos}

Os autores agradecem a CAPES pelo apoio financeiro e a Thornton Eletrônica Ltda pela doação recebida para os trabalhos experimentais em andamento.

\section{Referências}

AMA, Naji et al. PLL performance under frequency fluctuation-compliance with standards for distributed generation connected to the grid. 2013 IEEE PES Conference on Innovative Smart Grid Technologies, ISGT LA 2013 , 2013.

BACHA, Seddik; MUNTEANU, Iulian; BRATCU, Antoneta Iuliana. Power Electronic Converters Modeling and Control. Glasgow: Springer, 2014. 454 p.

BLAABJERG, Frede et al. Overview of Control and Grid Synchronization for Distributed Power Generation Systems. IEEE Transactions on Industrial Electronics v. 53, n. 5, p. 1398-1409, 2006.

BLASKO, Vladimir. A Novel Method for Selective Harmonic Elimination in Power Electronic Equipment. IEEE Transactions on Power Electronics v. 22, n. 1, p. 223 $228,2007$.

BOSIO, Federico De et al. Current control loop design and analysis based on resonant regulators for microgrid applications Current control loop design and analysis based on resonant regulators for microgrid applications. 2015, Yokohama: [s.n.], 2015. p. $5322-5327$. 
Técnicas de controle e métodos de sincronização para sistemas de energia renovável ligados à rede elétrica - Uma revisão

CASTILLA, Miguel et al. Control design guidelines for singlephase grid-connected photovoltaic inverters with damped resonant harmonic compensators. IEEE Transactions on Industrial Electronics v. 56, n. 11, p. 4492-4501, 2009.

CHEN, B.M. et al. Composite nonlinear feedback control for linear systems with input saturation: theory and an application. IEEE Transactions on Automatic Control v. 48, n. 3, p. 427-439 , 2003.

CHEN, Dong; ZHANG, Junming; QIAN, Zhaoming. An improved repetitive control scheme for grid-connected inverter with frequency-adaptive capability. IEEE Transactions on Industrial Electronics v. 60, n. 2, p. 814-823, 2013.

CIOBOTARU, Mihai; TEODORESCU, Remus; BLAABJERG, Frede. A new single-phase PLL structure based on second order generalized integrator. 2006, [S.1: s.n.], 2006.

COSTA-CASTELLÓ, Ramon; GRINÓ, Robert; FOSSAS, Enric. Odd-harmonic digital repetitive control of a single-phase current active filter. IEEE Transactions on Power Electronics v. 19, n. 4, p. 1060-1068, 2004.

DASH, Prajna Paramita; YAZDANI, Amirnaser. A Mathematical Model and Performance Evaluation for a SingleStage Grid-Connected Photovoltaic (PV) System. International Journal of Emerging Electric Power Systems v. 9, n. 6 , 2008.

DAVOODNEZHAD, Reza; HOLMES, Donald Grahame;MCGRATH, Brendan P. A Fully Digital Hysteresis Current Controller for Current Regulation of Grid Connected PV Inverters. 2014, [S.1: s.n.], 2014. p.24-27.

DESTRO, Rayra et al. Implementation aspects of adaptive window moving average filter applied to PLLs - Comparative study. 2013, [S.1: s.n.], 2013. p.730-736.

DUMITRESCU, A. M. et al. Design of current controllers for active power filters using naslin polynomial technique. 2007, [S.1: s.n.], 2007.
EREN, Suzan. Composite nonlinear feedback control and stability analysis of a grid-connected voltage source inverter with LCL filter. IEEE Transactions on Industrial Electronics v. 60, n. 11, p. $5059-5074,2013$.

EREN, Suzan; BAKHSHAI, Alireza; JAIN, Praveen. A Novel Nonlinear Current Control Technique for a GridConnected DC / AC Inverter used in Renewable Energy Power Conditioning Systems. 2014, [S.1: s.n.], 2014. p.1-8.

GAZOLI, Jonas Rafael et al. Microconversor integrado ao módulo fotovoltaico para sistemas conectados à rede elétrica utilizando controlador ressonante. Brazilian Journal of Power Electronics v. 18, n. 2, p. 908-916, 2013.

GOLESTAN, Saeed et al. An Efficient Implementation of Generalized Delayed Signal Cancellation PLL. IEEE Transactions on Power Electronics n. 99, p. 1-10 , 2015.

GOLESTAN, Saeed et al. PLL with MAF-Based Prefiltering Stage: Small-Signal Modeling and Performance Enhancement. IEEE Transactions on Power Electronics v. 31, n. 16 , p. $4013-4019,2016$.

GONZALEZ-ESPIN, Fran et al. An Adaptive Control System for Three-Phase Photovoltaic Inverters Working in a Polluted and Variable Frequency Electric Grid. IEEE Transactions on Power Electronics v. 27, n. 10, p. 4248$4261,2012$.

GONZALEZ-ESPIN, Fran et al. An Adaptive Digital Control Technique for Improved Performance of Grid Connected Inverters. IEEE Transactions on Industrial Informatics v. 9, n. 2, p. 708-718, 2013.

HO, Carl Ngai Man; CHEUNG, Victor S P; CHUNG, Henry Shu Hung. Constant-frequency hysteresis current control of grid-connected VSI without bandwidth control. IEEE Transactions on Power Electronics v. 24, n. 11, p. 2484$2495,2009$. 
KARIMI-GHARTEMANI, Masoud; IRAVANI, M. Reza. A method for synchronization of power electronic converters in polluted and variable-frequency environments. IEEE Transactions on Power Systems v. 19, n. 3, p. 1263-1270 , 2004.

LI, B. et al. Robust proportional resonant regulator for gridconnected voltage source inverter (VSI) using direct pole placement design method. IET Power Electronics v. 5, n. 8, p. 1367,2012 .

LIU, Tao et al. A novel repetitive control scheme for threephase grid-connected inverter with LCL filter. 2012, [S.1: s.n.], 2012. p.335-339.

MALESANI, Luigi. Robust dead-beat current control for PWM rectifiers and active filters. IEEE Transactions on Industry Applications v. 35, n. 3, p. 613-620, 1999.

MALESANI, Luigi; MATTAVELLI, Paolo. Improved constant-frequency hysteresis current control of VSI inverters with simple feedforward bandwidth prediction. IEEE Transactions on Industry Applications v. 33, n. 5, p. 1194-1202, 1997.

MASTROMAURO, R et al. A Single-Phase VoltageControlled Grid-Connected Photovoltaic System With Power Quality Conditioner Functionality. IEEE Transactions on Industrial Electronics v. 56, n. 11, p. 4436-4444, 2009.

MATTAVELLI, Paolo. An improved deadbeat control for UPS using disturbance observers. IEEE Transactions on Industrial Electronics v. 52, n. 1, p. 206-212, 2005.

MONFARED, Mohammad; GOLESTAN, Saeed. Control strategies for single-phase grid integration of small-scale renewable energy sources: A review. Renewable and Sustainable Energy Reviews v. 16, n. 7, p. 4982-4993 , 2012.

NASCIMENTO, Paulo Sergio et al. Design methodology of P-res controllers with harmonic compensation for threephase DC-AC grid-tie inverters with LCL output filter. 2014, Santander: [s.n.], 2014. p.1-8.
NAZIR, Rabia et al. Frequency adaptive repetitive control of grid-connected inverters. 2014 International Conference on Control, Decision and Information Technologies (CoDIT) $n$. November, p. 584-588, 2014.

NEWMAN, Michael John; HOLMES, Donald Grahame. Delta operator digital filters for high performance inverter applications. IEEE Transactions on Power Electronics v. 18, n. 1 II, p. 447-454, 2003.

PÁDUA, M. S. et al. Comparative analysis of synchronization algorithms based on PLL, RDFT and Kalman Filter. IEEE International Symposium on Industrial Electronics n. 1, p. 964-970.

PARKER, Stewart Geoffrey; MCGRATH, Brendan P.; HOLMES, Donald Grahame. Regions of active damping control for LCL filters. IEEE Transactions on Industry Applications v. 50, n. 1, p. 424-432, 2014.

RODRÍGUEZ, Pedro et al. Decoupled double synchronous reference frame PLL for power converters control. IEEE Transactions on Power Electronics v. 22, n. 2, p. 584-592 2007.

SACCOMANDO, Giuseppe; SVENSSON, Jan. Transient Operation of Grid-connected Voltage Source Converter Under Unbalanced Voltage Conditions. 2001, Chicago: [s.n.], 2001. p.2419-2424.

SERA, Dezso et al. Low-cost digital implementation of proportional-resonant current controllers for PV inverter applications using delta operator. 2005, [S.1: s.n.], 2005. p.2517-2522.

SILVA, Sergio A O et al. PLL Structures for Utility Connected Systems under Distorted Utility Conditions. 2006, Paris: [s.n.], 2006. p.2636-2641.

SUUL, J A et al. Impedance-compensated grid synchronization for extending the stability range of weak grids with voltage source converters. IET Generation, Transmission \& Distribution p. 1-12, 2015. 
Técnicas de controle e métodos de sincronização para sistemas de energia renovável ligados à rede elétrica - Uma revisão

SUUL, Jon Are et al. Synchronous Reference Frame Hysteresis Synchronous Reference Frame Hysteresis Current Control for Grid Converter Applications. IEEE Transactions on Industry Applications v. 47, n. 5, p. 2183-2194, 2011.

TEODORESCU, Remus et al. Proportional-resonant controllers and filters for grid-connected voltage-source converters. 2006, [S.1: s.n.], 2006. p.750-762.

TEODORESCU, Remus; BLAABJERG, Frede. A New Control Structure for Grid-Connected LCL PV Inverters with Zero Steady-State Error and Selective Harmonic Compensation. 2004a, [S.1: s.n.], 2004. p.580-586.

TEODORESCU, Remus; BLAABJERG, Frede. Proportionalresonant controllers. A new breed of controllers suitable for grid-connected voltage-source converters. 2004b, Brasov: [s.n.], 2004. p.9-14.

TEODORESCU, Remus; LISERRE, Marco; RODRIGUEZ, P. Grid converters for photovoltaic and wind power systems. Londres: JohnWiley and Sons, Ltd, 2011. 407 p.

TIMBUS, A et al. Evaluation of Current Controllers for Distributed Power Generation Systems. Power Electronics, IEEE Transactions on v. 24, n. 3, p. 654-664, 2009.

TWINING, Erika; HOLMES, Donald Grahame. Grid current regulation of a three-phase voltage source inverter with an LCL input filter. IEEE Transactions on Power Electronics v. 18, n. 3, p. 888-895, 2003.

WU, Xiaojie et al. Grid Harmonics Suppression Scheme for LCL -Type Grid-Connected Inverters Based on Output Admittance Revision. IEEE Transactions on Sustainable Energy v. 6, n. 2, p. $411-421,2015$.

YANG, Y; ZHOU, K; BLAABJERG, F. Enhancing the Frequency Adaptability of Periodic Current Controllers with a Fixed Sampling Rate for Grid-Connected Power Converters. IEEE Transactions on Power Electronics v. 31, n. 10, p. $7273-7285,2016$.
YANG, Yongheng. Advanced Control Strategies to Enable a More Wide-Scale Adoption of Single-Phase Photovoltaic Systems. 2014.

YANG, Yongheng et al. Suggested Grid Code Modifications to Ensure Wide-Scale Adoption of Photovoltaic Energy in Distributed Power Generation Systems. 2013, [S.1: s.n.], 2013. p.1-8.

YANG, Yongheng; WANG, Huai; BLAABJERG, Frede. Reactive power injection strategies for single-phase photovoltaic systems considering grid requirements. 2014, [S.1: s.n.], 2014. p.371-378.

YANG, Yongheng; ZHOU, Keliang; BLAABJERG, Frede. Frequency Adaptability of Harmonics Controllers for GridInterfaced Converters. International Journal of Control p. $1-12,2015$.

YEPES, Alejandro G. et al. Analysis and design of resonant current controllers for voltage-source converters by means of nyquist diagrams and sensitivity function. IEEE Transactions on Industrial Electronics v. 58, n. 11, p. 5231-5250, 2011a.

YEPES, Alejandro G. et al. Effects of discretization methods on the performance of resonant controllers. IEEE Transactions on Power Electronics v. 25, n. 7, p. 1692-1712 , 2010 .

YEPES, Alejandro G. et al. Evaluation of techniques for cross-coupling decoupling between orthogonal axes in double synchronous reference frame current control. IEEE Transactions on Industrial Electronics v. 61, n. 7, p. 3527$3531,2014$.

YEPES, Alejandro G. et al. High-performance digital resonant controllers implemented with two integrators. IEEE Transactions on Power Electronics v. 26, n. 2, p. 563-576, 2011b.

YUAN, Xiaoming et al. Stationary-frame generalized integrators for current control of active power filters with zero steady-state error for current harmonics of concern under unbalanced and distorted operating conditions. IEEE Transactions on Industry Applications v. 38, n. 2, p. 523-532 , 2002. 
ZANCHETTA, Pericle; SHARKH, Suleiman; ABUSARA, Mohammad. Adaptive repetitive control with feedforward scheme for grid-connected inverters. IET Power Electronics v. 8, n. 8, p. $1403-1410,2015$.

ZHANG, Xiaotian; SPENCER, Joseph W.; GUERRERO, Josep M. Small-signal modeling of digitally controlled gridconnected inverters with LCL filters. IEEE Transactions on Industrial Electronics v. 60, n. 9, p. 3752-3765, 2013.

ZHOU, Sizhan et al. Cross-coupling and Decoupling Techniques in the Current Control of Grid-connected Voltage Source Converter. 2015, [S.1: s.n.], 2015. p.2821-2827. ZMOOD, Daniel Nahum; HOLMES, Donald Grahame. Stationary Frame Current Regulation of PWM Inverters With Zero Steady-State Error. IEEE Transactions on Power Electronics v. 18, n. 3, p. 814-822, 2003.

Recebido em 2 Dezembro, 2015 - Received on

December 2, 2015 Aceito em 29 Setembro, 2016 - Accepted on September 29, 2016 